\section{(2) \\ BRAZIULIAN JOURNAL \\ OF MEDICAL AND BIOLOGICAL RESEARCH}

www.bjournal.com.br
ISSN 0100-879X

Volume 45 (7) 565-680

July 2012

CLINICAL INVESTIGATION

Braz J Med Biol Res, July 2012, Volume 45(7) 610-616

doi: 10.1590/S0100-879X2012007500083

Hemodynamic mechanisms of the attenuated blood pressure response to mental stress after a single bout of maximal dynamic exercise in healthy subjects

F.J. Neves, A.C.G. Carvalho, N.G. Rocha, B.M. Silva, A.R.K. Sales, R.R.T. de Castro,

J.D. Rocha, T.G. Thomaz and A.C.L. Nóbrega

The Brazilian Journal of Medical and Biological Research is partially financed by

\section{Q}

da Ciência e Tecnologia

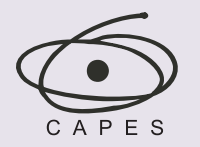

Ministério da Educação

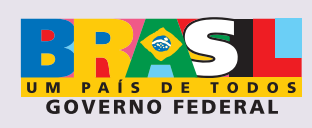

DTFAPESP

Institutional Sponsors

Ф SHIMADZU UNICAMP

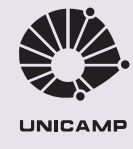

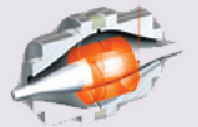

1D. Associaçăo Explore High - Performance MS Orbitrap Technology In Proteomics \& Metabolomics analitica $\underset{\text { analiticaweb.com.br }}{\text { Thermo }}$ S I E N I FIC
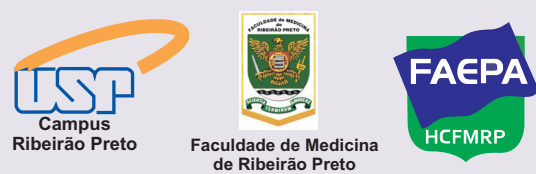


\title{
Hemodynamic mechanisms of the attenuated blood pressure response to mental stress after a single bout of maximal dynamic exercise in healthy subjects
}

\author{
F.J. Neves ${ }^{1}$, A.C.G. Carvalho ${ }^{1}$, N.G. Rocha ${ }^{1}$, B.M. Silva ${ }^{1}$, A.R.K. Sales ${ }^{1}$, \\ R.R.T. de Castro ${ }^{1}$, J.D. Rocha ${ }^{1}$, T.G. Thomaz ${ }^{2}$ and A.C.L. Nóbrega ${ }^{1,2}$ \\ ${ }^{1}$ Programa de Pós-Graduação em Ciências Cardiovasculares, Faculdade de Medicina, \\ Universidade Federal Fluminense, Niterói, RJ, Brasil \\ ${ }^{2}$ Departamento de Fisiologia e Farmacologia, Instituto Biomédico, \\ Universidade Federal Fluminense, Niterói, RJ, Brasil
}

\begin{abstract}
To determine the hemodynamic mechanisms responsible for the attenuated blood pressure response to mental stress after exercise, 26 healthy sedentary individuals (age $29 \pm 8$ years) underwent the Stroop color-word test before and 60 min after a bout of maximal dynamic exercise on a treadmill. A subgroup $(\mathrm{N}=11)$ underwent a time-control experiment without exercise. Blood pressure was continuously and noninvasively recorded by infrared finger photoplethysmography. Stroke volume was derived from pressure signals, and cardiac output and peripheral vascular resistance were calculated. Perceived mental stress scores were comparable between mental stress tests both in the exercise $(P=0.96)$ and control $(P=0.24)$ experiments. After exercise, the systolic blood pressure response to mental stress was attenuated (pre: $10 \pm 13$ vs post: $6 \pm 7 \mathrm{mmHg}$; $\mathrm{P}<0.01$ ) along with lower values of systolic blood pressure (pre: $129 \pm 3$ vs post: $125 \pm 3 \mathrm{mmHg}$; $\mathrm{P}<0.05$ ), stroke volume (pre: 89.4 \pm 3.5 vs post: $76.8 \pm 3.8 \mathrm{~mL} ; \mathrm{P}<0.05$ ), and cardiac output (pre: $7.00 \pm 0.30$ vs post: $6.51 \pm 0.36 \mathrm{~L} / \mathrm{min} ; \mathrm{P}<0.05$ ). Except for heart rate, the hemodynamic responses and the mean values during the two mental stress tests in the control experiment were similar $(P>0.05)$. In conclusion, a single bout of maximal dynamic exercise attenuates the blood pressure response to mental stress in healthy subjects, along with lower stroke volume and cardiac output, denoting an acute modulatory action of exercise on the central hemodynamic response to mental stress.
\end{abstract}

Key words: Cardiovascular system; Blood pressure; Exercise test; Stroop test

\section{Introduction}

Mentally and physically stressful situations occur frequently throughout our lives and the capacity to respond to them, both in motor and physiological terms, are natural and necessary organic functions. The cardiovascular system is directly involved in the response to mental stress, thus suffering in exacerbated stressful conditions. During mental stress, sympathetic nervous system activation and parasympathetic inhibition increase arterial pressure and heart rate, leading to increased myocardial oxygen consumption (1). Although this is a physiological phenomenon, exacerbated responses of blood pressure confer an increased risk of developing hypertension $(2,3)$.
Moreover, susceptible individuals may present a reduction of myocardial perfusion and increased electrical instability during stress, triggering acute myocardial infarction and cardiac arrhythmias $(4,5)$.

Physical exercise promotes complex physiological responses of the cardiovascular system, which are dependent on the interaction between cardiac, vascular, and autonomic mechanisms, responsible for the maintenance of homeostasis $(6,7)$. An essential hemodynamic response is the increased cardiac output and its redistribution brought about by the combination of metabolic vasodilatation in active muscles and adrenergic neural vasoconstriction in the

Correspondence: A.C.L. Nóbrega, Departamento de Fisiologia e Farmacologia, IB, UFF, Rua Professor Hernani Pires de Mello, 101, Sala 106, 24210-130 Niterói, RJ, Brasil. Fax: +55-21-2629-2404. E-mail: anobrega@id.uff.br

Received January 20, 2012. Accepted May 3, 2012. Available online May 18, 2012. Published July 2, 2012. 
splanchnic bed and resting muscles $(8,9)$. The physiological impact of exercise persists for hours after cessation of a single exercise bout (7). Whereas some variables that increase or decrease during exercise (such as heart rate and systemic vascular resistance, respectively) show a progressive return to baseline values during the recovery period, others may exhibit an overcompensation leading to values that can remain lower or higher than before exercise for minutes or hours (7). The temporal summation of these effects is believed to be at least part of the mechanism responsible for developing the chronic adaptations of exercise training (7), which are well known to decrease the risk of developing chronic diseases (10). Therefore, the investigation of the physiological mechanisms involved in the hemodynamic responses after a single bout of exercise could contribute to the understanding of the process of decreasing the risk of chronic diseases as a result of physical training.

It is well known that a single bout of exercise can produce, under some circumstances, a hypotensive effect that may last several hours, a phenomenon usually named post-exercise hypotension $(7,11)$. Several factors, such as resting blood pressure values, exercise intensity, muscle mass, and body position among others (12-15), determine the magnitude of the phenomenon, or even whether or not the phenomenon occurs. Even when blood pressure does not decrease below pre-exercise values, the autonomic and hemodynamic functions remain altered for several minutes or hours after a single bout of exercise $(7,11,16)$. Thus, it is not surprising that the blood pressure response to mental stress, which is of relevant prognostic value $(2,3)$, is attenuated after a single bout of exercise (17-21). Since blood pressure is the result of the relationship between cardiac output and systemic vascular resistance, either vascular (peripheral) or cardiac (central) mechanisms could be responsible for the attenuation of the blood pressure response observed after an exercise bout, but these hemodynamic mechanisms have not been specifically studied.

Previous publications have shown that resting cardiac output is reduced after a single bout of exercise $(16,22)$ and that stroke volume tends to decrease during mental stress (23). On the other hand, peripheral vascular resistance (PVR) does not change or may even increase (24). Considering this background, we hypothesized that the smaller increase of blood pressure during mental stress after a single bout of dynamic exercise would occur by a central mechanism, i.e., by a reduced cardiac output.

Therefore, the aim of the present study was to investigate the effects of a single bout of maximal dynamic exercise on the cardiovascular responses to mental stress in healthy young sedentary individuals in order to investigate the hemodynamic components responsible for the reduced blood pressure response in these conditions.

\section{Subjects and Methods}

\section{Subjects}

A group of 26 healthy sedentary volunteers (15\% men; age $29 \pm 8$ years) were recruited. The eligibility for taking part in the study was based on the assessment of clinical history and physical examination, blood pressure measurement, biochemical blood analyses, resting electrocardiogram, and maximal cardiopulmonary exercise testing. All subjects fulfilled the following criteria: age between 18 and 49 years, women with regular menstrual cycles, absence of any diagnosed disease and any recent infections, body mass index between 18.5 and $29.9 \mathrm{~kg} / \mathrm{m}^{2}$, systolic blood pressure $<140 \mathrm{mmHg}$ and/or diastolic blood pressure $<90 \mathrm{mmHg}$, glycemia $<100 \mathrm{mg} / \mathrm{dL}$, total cholesterol $<240$ $\mathrm{mg} / \mathrm{dL}$, low-density lipoprotein cholesterol $<160 \mathrm{mg} / \mathrm{dL}$, triglycerides $<200 \mathrm{mg} / \mathrm{dL}$, non-smoker, not using medications with the exception of oral contraceptives, normal resting and exercise electrocardiogram, and sedentary (subjects were not engaged in physical activities lasting $30 \mathrm{~min}$ or more of moderate/severe intensity, three times per week during the last 3 months). The study procedures were approved by the Ethics Committee of Hospital Universitário Antonio Pedro (CEP-CCM/HUAP 005/07), and written informed consent was obtained from all participants prior to the experimental procedures.

\section{Experimental protocol}

After the initial clinical and laboratory screening, experiments were conducted in the morning $1 \mathrm{~h}$ after a standardized light breakfast. Women were evaluated between the 1 st and the 12th day of the menstrual cycle. Participants did not drink alcohol or caffeinated beverages and did not perform intense physical activity for at least $24 \mathrm{~h}$ before the visits. Subjects were placed in the supine position in a quiet air-conditioned room (approximately $24^{\circ} \mathrm{C}$ ) and rested quietly for $10 \mathrm{~min}$. The experimental protocol consisted of measurements of blood pressure response by beat-to-beat finger infrared plethysmography (see below) to a mental stress challenge both before and $1 \mathrm{~h}$ after a single bout of maximal dynamic exercise (see below).

A subgroup of 11 subjects also participated in a timecontrol protocol, which was conducted on a day other than that of the experimental protocol. These were the last 11 consecutive subjects enrolled in the study and this subgroup was of similar gender ( $18 \%$ men) and age ( $28 \pm 7$ years) as the rest of the sample. The order of the control and experimental protocols was randomized in this subgroup. The control protocol consisted of measurements of blood pressure response to a mental stress challenge both before and $1 \mathrm{~h}$ after standing on a treadmill for 30 min without exercising, which was the approximate duration of the whole exercise bout procedure. Therefore, any difference in the test-retest changes between groups would be interpreted as being a specific effect of the exercise bout, and not just 
an accommodation effect of performing two subsequent mental stress tests.

\section{Measurements}

Systemic hemodynamics. Noninvasive beat-to-beat blood pressure values were monitored continuously at baseline and during the mental stress by finger photoplethysmography (Finometer ${ }^{\circledR}$, Finapres Medical Systems, The Netherlands). A cuff was placed on the left middle finger for a beat-by-beat blood pressure assessment. The heart rate at baseline and during the mental stress was automatically calculated from the intervals between peak systolic values. Stroke volume was derived from pressure signals, and cardiac output and PVR were calculated using the Modelflow method (25), which is an automatic method that computes aortic blood flow from the arterial pressure wave by simulating a nonlinear, time-varying three-element model of aortic input impedance, and incorporates in the calculation data related to gender, age, height, and body weight (BeatScope 1.0 software, Finapres Medical Systems). Finger photoplethysmography data were recorded continuously for $5 \mathrm{~min}$ at baseline and during the first minute of mental stress, and the average of the recorded period was used for analysis. The signal was edited by applying an automatic generalized waveform filter, and differences in pressure level were corrected by a generalized level correction equation using filtered systolic and diastolic levels (BeatScope 1.0 software, Finapres Medical Systems) (26).

Mental stress. The mental stress challenge was induced through a computerized version of the Stroop word-color conflict test (27) in the course of $3 \mathrm{~min}$. The test consisted of a PowerPoint presentation on a computer monitor placed in front of the subjects. The slides changed automatically each $2 \mathrm{~s}$, and the test was performed with continuous auditory conflict. The subjects had to tell, as quickly as possible, the color of the letters presented on the slides. At the end of the test the subjects were asked about the level of difficulty of the test according to the following perceived mental stress score: $0=$ non-stressful, 1 = slightly stressful, 2 = stressful, 3 = very stressful, and 4 = extremely stressful (28).

Single bout of maximal dynamic exercise. A maximal cardiopulmonary exercise test was performed on a treadmill (Super ATL, Inbrasport, Brazil). The test consisted of a 3-min warm-up at $3 \mathrm{~km} / \mathrm{h}$ and $0 \%$ grade, followed by a ramp protocol with linear increase of speed and grade, and by a 5 -min recovery phase at $4 \mathrm{~km} / \mathrm{h}$ and $0 \%$ grade. The ramp protocol was individualized according to the predicted maximal exercise capacity to reach volitional fatigue at approximately $10 \mathrm{~min}$. Subjects were verbally encouraged to exercise until exhaustion. All subjects met at least two of the following criteria to confirm that maximal effort was attained: 1$)$ respiratory exchange ratio $>1.1 ; 2)$ heart rate within \pm 10 bpm of the age-predicted maximum [210 - (age $x$
0.65)]; 3) score 10 of perceived effort on the 0 -10 Borg scale. Ventilation, oxygen uptake and carbon dioxide output were measured with each breath (CPX Ultima Gas Exchange System, Medgraphics, USA). Electrocardiograms were monitored through 12 leads (CardioPerfect ${ }^{\mathrm{TM}}$ Workstation, Welch Allyn, USA) and perceived exertion was assessed every minute through the $0-10$ Borg scale.

\section{Statistical analysis}

The Shapiro-Wilk test and the Levene test were used to verify the distribution of the variables and variance homogeneity, respectively. Geisser-Greenhouse correction was used for repeated measure analysis to correct for violations of the sphericity assumption. Two-way ANOVA was followed by the Bonferroni post hoc test to compare the measurements at baseline and during the mental stress (factor 1), before and after exercise (factor 2). Anthropometric, metabolic and hemodynamic characteristics of the exercise and control groups were compared by the unpaired Student $t$-test, whereas the perceived mental stress score was compared by the nonparametric Wilcoxon test. This same nonparametric test was employed to compare the hemodynamic responses (measured as both absolute and percent changes) to the mental stress test performed before and after exercise (experimental group) and to compare the hemodynamic responses to the first and the second mental stress test (control group). The sample size was calculated using the percent change of systolic blood pressure as the main outcome, considering a difference of $25 \%$ between the pre- and post-exercise values and a standard deviation of $20 \%$. These values were obtained in pilot studies from our laboratory and from data published by other groups (18). This analysis showed that a sample size of 9 subjects would reach statistical significance for an alpha of 0.05 and power ( 1 - beta) of 0.80 in a paired Student $t$-test. Values are reported as means \pm SEM when are normally distributed or as median (quartile range) when the variable was not normally distributed. Statistical significance was considered to be $\mathrm{P}<0.05$. All analyses were performed with the Statistica software (version 8, StatSoft, Inc., USA).

\section{Results}

Anthropometric, metabolic and hemodynamic characteristics of the subjects are presented in Table 1. There were no differences between the subjects included in the single bout of maximal dynamic exercise or in the control non-exercise experiment $(P>0.05)$. The perceived mental stress score was similar at pre- and post-intervention in the exercise and control experiments (exercise experiment: pre $=1.8 \pm 1.0 \mathrm{vs}$ post $=1.7 \pm 1.0, \mathrm{P}=0.96$; control experiment: pre $=1.6 \pm 1.0$ vs post $=1.6 \pm 1.1, \mathrm{P}=0.24)$.

Mental stress increased heart rate, systolic, mean and diastolic blood pressure, as well as cardiac output when 
performed before exercise, while stroke volume and PVR did not change (Table 2). After exercise, baseline mean blood pressure, diastolic blood pressure, and heart rate were higher $(P<0.05)$, whereas stroke volume was lower $(P<0.05)$ than before exercise (Table 2$)$. When the mental stress test was performed after the exercise bout, it caused similar hemodynamic responses as before exercise, including increased heart rate, systolic, mean, and diastolic blood pressure, and cardiac output (Table 2). However, systolic blood pressure, stroke volume and cardiac output reached lower absolute values despite the higher heart rate (Table 2).

The systolic blood pressure response to mental stress, measured as absolute (10 \pm 13 vs $6 \pm 7 \mathrm{mmHg}$; $\mathrm{P}<0.01)$ and percent ( $9 \pm 12$ vs $5 \pm 5 \% ; P=0.03$ ) changes, was attenuated after exercise, but it was not different in the control group ( $7 \pm 19$ vs $4 \pm 5 \mathrm{mmHg}, \mathrm{P}>0.05 ; 5 \pm 21$ vs $3 \pm 5 \%, P>0.05)$. Besides the attenuated blood pressure response to mental stress after exercise (see above), no other variable presented significantly different responses (changes) before or after exercise $(P>0.05)$ : heart rate: pre $=11 \pm 17$ vs post $=9 \pm 8 \mathrm{bpm}$; stroke volume: pre $=$ $-1.2 \pm 9.0$ vs post $=-3.0 \pm 7.0 \mathrm{~mL}$; cardiac output: pre $=$ $-0.5 \pm 1.6$ vs post $=-0.4 \pm 1.1 \mathrm{~L} / \mathrm{min}$; PVR: pre $=-0.02 \pm$ 0.16 vs post $=-0.07 \pm 0.14 \mathrm{AU}$.
Table 1. Anthropometric, metabolic, and hemodynamic characteristics of the subjects studied.

\begin{tabular}{lcc}
\hline Variables & Exercise & Control \\
\hline N (\% men) & $26(15 \%)$ & $11(18 \%)$ \\
Age (years) & $29 \pm 8$ & $28 \pm 2$ \\
Weight $(\mathrm{kg})$ & $68.5 \pm 2.2$ & $66.5 \pm 3.5$ \\
Body mass index $\left(\mathrm{kg} / \mathrm{m}^{2}\right)$ & $25.1 \pm 0.7$ & $24.1 \pm 1.1$ \\
Fasting glucose $(\mathrm{mg} / \mathrm{dL})$ & $85.2 \pm 2.0$ & $84.5 \pm 3.6$ \\
Cholesterol $(\mathrm{mg} / \mathrm{dL})$ & $163.4 \pm 5.5$ & $163.4 \pm 7.2$ \\
HDL cholesterol $(\mathrm{mg} / \mathrm{dL})$ & $53.8 \pm 2.9$ & $51.4 \pm 4.1$ \\
LDL cholesterol $(\mathrm{mg} / \mathrm{dL})$ & $90.9 \pm 5.4$ & $90.3 \pm 8.2$ \\
Triglycerides $(\mathrm{mg} / \mathrm{dL})$ & $93.8 \pm 6.7$ & $108.3 \pm 11.5$ \\
Systolic blood pressure $(\mathrm{mmHg})$ & $113 \pm 3$ & $110 \pm 4$ \\
Diastolic blood pressure $(\mathrm{mmHg})$ & $72 \pm 2$ & $70 \pm 3$ \\
Mean blood pressure $(\mathrm{mmHg})$ & $86 \pm 2$ & $83 \pm 3$ \\
Resting heart rate $(\mathrm{bpm})$ & $68 \pm 2$ & $68 \pm 2$ \\
Peak heart rate $(\mathrm{bpm})$ & $175 \pm 4$ & $180 \pm 5$ \\
Peak oxygen uptake $\left(\mathrm{mL} \cdot \mathrm{kg}^{-1} \cdot \mathrm{min}^{-1}\right)$ & $30.2 \pm 1.5$ & $30.6 \pm 2.8$ \\
\hline
\end{tabular}

Data are reported as means \pm SEM, except for the number $(N)$ of subjects. $\mathrm{HDL}=$ high-density lipoprotein; $\mathrm{LDL}=$ low-density lipoprotein. There were no differences between groups $(P>0.05$, Student $t$-test).

Table 2. Hemodynamic variables during a mental stress test performed before and after a single bout of maximal dynamic exercise $(\mathrm{N}=26)$ or during a control experiment involving no exercise $(\mathrm{N}=11)$.

\begin{tabular}{lccccc}
\hline Variables & Baseline & Mental stress & & Baseline & Mental stress \\
\hline Exercise & \multicolumn{2}{c}{ Pre-exercise } & & \multicolumn{2}{c}{ Post-exercise } \\
\cline { 2 - 3 } \cline { 5 - 6 } Systolic blood pressure (mmHg) & $117 \pm 2$ & $129 \pm 3^{*}$ & & $120 \pm 3$ & $125 \pm 3^{*+}$ \\
Mean arterial blood pressure (mmHg) & $86 \pm 2$ & $96 \pm 3^{*}$ & & $90 \pm 3^{+}$ & $95 \pm 3^{*}$ \\
Diastolic blood pressure (mmHg) & $68 \pm 2$ & $77 \pm 2^{*}$ & & $72 \pm 2^{+}$ & $78 \pm 2^{*}$ \\
Heart rate (bpm) & $67 \pm 2$ & $80 \pm 3^{*}$ & & $75 \pm 2^{+}$ & $86 \pm 3^{*+}$ \\
Stroke volume (mL) & $91.4 \pm 3.1$ & $89.4 \pm 3.5$ & & $78.9 \pm 3.4^{+}$ & $76.8 \pm 3.8^{+}$ \\
Peripheral vascular resistance (AU) & $0.91 \pm 0.03$ & $0.90 \pm 0.04$ & & $1.01 \pm 0.06$ & $0.98 \pm 0.07$ \\
Cardiac output (L/min) & $6.08 \pm 0.19$ & $7.00 \pm 0.30^{*}$ & & $5.86 \pm 0.24$ & $6.51 \pm 0.36^{*+}$ \\
Control & \multicolumn{2}{c}{ Pre-control } & & & Post-control \\
Systolic blood pressure (mmHg) & $114 \pm 5$ & $125 \pm 6^{*}$ & & $117 \pm 5$ & $121 \pm 5^{*}$ \\
Diastolic blood pressure (mmHg) & $67 \pm 3$ & $75 \pm 4^{*}$ & & $69 \pm 4$ & $73 \pm 4^{*}$ \\
Mean arterial blood pressure (mmHg) & $87 \pm 4$ & $96 \pm 5^{*}$ & & $89 \pm 4$ & $94 \pm 4^{*}$ \\
Heart rate (bpm) & $68 \pm 2$ & $77 \pm 3^{*}$ & & $63 \pm 2$ & $68 \pm 3^{+}$ \\
Stroke volume (mL) & $85.6 \pm 6.5$ & $83.4 \pm 6.8$ & & $82.6 \pm 5.6$ & $79.5 \pm 5.2$ \\
Peripheral vascular resistance (AU) & $0.96 \pm 0.07$ & $0.97 \pm 0.09$ & & $1.09 \pm 0.08$ & $1.11 \pm 0.09$ \\
Cardiac output (L/min) & $5.77 \pm 0.48$ & $6.42 \pm 0.65$ & & $5.13 \pm 0.32$ & $5.27 \pm 0.25$ \\
\hline
\end{tabular}

Data are reported as means \pm SEM. ${ }^{*} P<0.01 v s$ baseline within the pre- or post-exercise period. ${ }^{+} \mathrm{P}<0.05 v s$ corresponding measurement at pre-exercise (two-way ANOVA and Bonferroni test). 


\section{Discussion}

The physiological after-effects after a single bout of exercise may have an excitatory or an inhibitory profile, i.e., an increase or decrease of the magnitude of different variables after exercise (7). The altered physiological environment created by the exercise bout may persist for hours and is able to modulate the pressor response to a subsequent mental stress challenge, an effect observed in the present experiment as well as in previous publications $(19,22)$. In the present study, resting blood pressure was not lower after exercise, denoting the absence of post-exercise hypotension. Post-exercise hypotension is a variable phenomenon that depends on several different factors such as intensity and duration of the exercise, previous blood pressure values (13), body position (12), and muscle mass (14), or may not even occur $(15,29)$. In the subjects involved in the present study this phenomenon was not observed, probably because of the characteristics of the exercise bout, i.e., progressive dynamic exercise to exhaustion of about 10-min duration. Although this type of exercise has been shown to increase vascular reactivity in healthy individuals (30), more prolonged periods of exercise seem to cause a more pronounced drop in resting blood pressure (19).

Regardless of the absence of post-exercise hypotension itself, both the mean absolute values and the response to mental stress were attenuated. Thus, the effect of a single bout of exercise represented an attenuated pressure reactivity per se during mental stress, since the magnitude of the pressure changes was lower when the mental stress test was performed after the physical effort. This is in accordance with a meta-analysis that concluded that a single bout of exercise causes a consistent attenuation of the blood pressure response to a psychosocial stressor (19). It is still largely unknown which combination of exercise mode, intensity and duration causes the greatest reduction of cardiovascular reactivity. Nevertheless, attenuation of the pressure response to mental stress seems to be evident in submaximal exercise bouts lasting at least $30 \mathrm{~min}$ at $50 \%$ of maximal oxygen consumption, with higher intensities tending to show greater effects $(19,31)$. The present study expands this concept showing that dynamic progressive exercise at maximal intensity also leads to reduced pressure reactivity in mental stress tests performed $1 \mathrm{~h}$ after a single bout of peak exercise.

Acomplex mechanism modulates the interplay between the various components of the cardiovascular system in the regulation of blood pressure that is a function of cardiac output and PVR. Therefore, both central (cardiac) and peripheral (vascular) responses to mental stress interact to cause an increase in blood pressure, but few studies have attempted to examine the physiological mechanisms of the attenuated blood pressure response to mental stress after exercise $(18,19,23,32)$. In subjects with prehypertension, a mental stress test applied after a single bout of exercise provoked an attenuated blood pressure response along with enhanced forearm blood flow and conductance (32). In a different study, subjects with a family history of hypertension exhibited an attenuated increase in forearm blood flow induced by mental stress (23). However, these changes in forearm blood flow do not reflect the overall circulation, since total peripheral resistance does not change (23). The mechanism underlying the attenuated blood pressure response to mental stress after exercise seems to involve a decrease in sympathetic drive identified by a reduced plasma norepinephrine response and elongation of the pre-ejection period measured by thoracic impedance (18).

In the present study, the lower blood pressure response during mental stress after exercise occurred with no significant changes in the response of either cardiac output or PVR. However, resting values of stroke volume and mean values of both stroke volume and cardiac output during mental stress were lower after exercise. On the other hand, there were no differences between pre- and post-exercise values during resting or mental stress for PVR. Therefore, it seems that the exercise bout led to decreased levels of stroke volume both at rest and during mental stress, which in turn limited the increase in cardiac output during mental stress.

It has been previously shown that resting cardiac output is reduced after a single bout of submaximal dynamic exercise $(16,22,24)$. In the present study, cardiac output reached a lower value during mental stress performed after exercise, an effect that occurred along with a lower stroke volume and despite a higher heart rate. These lower values of stroke volume at baseline and during mental stress after maximal exercise were probably due to a reduction in cardiac preload that might have been caused by venodilation and/or diminished plasma volume, or a combination of both $(8,9,16,33)$.

Although the present study was not designed to investigate clinical outcomes, it is tempting to speculate that the attenuating effect of a single bout of exercise on the pressure response to mental stress may be summed along time to create a condition of lower risk for cardiovascular events. Previous studies have reported a close relationship between increased pressure responses to mental stress and increased risk to develop hypertension $(2,34)$. In this context, cardiovascular reactivity tests have been proposed as clinical tools for estimating the risk to develop hypertension (35). In addition, exercise training produces a plethora of physiological adaptations, among them an attenuated blood pressure response to mental stress (36), suggesting that the modulatory effect of an exercise bout described in the present study indeed sums up with a subsequent stimulus to develop the chronic effect of training (7).

\section{Limitations of the study}

The exercise protocol used (maximal exercise bout) 
is not frequently encountered in daily life. However, the maximal dynamic exercise test is a standardized protocol used to evaluate integrative cardiovascular, respiratory and muscular functions and health [Statement from the American Heart Association $(37,38)]$. In addition, previous studies from our group revealed that after a maximal exercise test, which is a well-standardized protocol, healthy subjects showed increased vascular reactivity (30). Based on this previous observation, we chose to use the maximum stress test, since the purpose of this study was to investigate the hemodynamic mechanisms responsible for lowering the blood pressure during mental stress. Indeed, it would be interesting if future studies advance the knowledge about the effect of exercise, especially regarding volume and intensity. Nevertheless, the present model provides the opportunity to investigate the impact of a maximal physiological challenge (peak exercise) on another phenomenon (response to mental stress), which was the main purpose of the study.

In conclusion, the blood pressure response to mental

\section{References}

1. Boutcher YN, Boutcher SH. Cardiovascular response to Stroop: effect of verbal response and task difficulty. Biol Psychol 2006; 73: 235-241.

2. Matthews KA, Katholi CR, McCreath H, Whooley MA, Williams DR, Zhu S, et al. Blood pressure reactivity to psychological stress predicts hypertension in the CARDIA study. Circulation 2004; 110: 74-78.

3. Armario P, del Rey RH, Martin-Baranera M, Almendros MC, Ceresuela LM, Pardell $\mathrm{H}$. Blood pressure reactivity to mental stress task as a determinant of sustained hypertension after 5 years of follow-up. J Hum Hypertens 2003; 17: 181-186.

4. Legault SE, Freeman MR, Langer A, Armstrong PW. Pathophysiology and time course of silent myocardial ischaemia during mental stress: clinical, anatomical, and physiological correlates. Br Heart J 1995; 73: 242-249.

5. Zotti AM, Bettinardi O, Soffiantino F, Tavazzi L, Steptoe A. Psychophysiological stress testing in postinfarction patients. Psychological correlates of cardiovascular arousal and abnormal cardiac responses. Circulation 1991; 83: II-25-II-35.

6. Harris KF, Matthews KA. Interactions between autonomic nervous system activity and endothelial function: a model for the development of cardiovascular disease. Psychosom Med 2004; 66: 153-164.

7. da Nobrega AC. The subacute effects of exercise: concept, characteristics, and clinical implications. Exerc Sport Sci Rev 2005; 33: 84-87.

8. Joyner MJ. Feeding the sleeping giant: muscle blood flow during whole body exercise. J Physiol 2004; 558: 1.

9. Joyner MJ, Wilkins BW. Exercise hyperaemia: is anything obligatory but the hyperaemia? J Physiol 2007; 583: 855860.

10. Cuffe M. The patient with cardiovascular disease: treatment strategies for preventing major events. Clin Cardiol 2006; 29: II-4-II-12.

11. Halliwill JR. Mechanisms and clinical implications of post- stress is attenuated after a single bout of maximal dynamic exercise in healthy subjects, an effect that occurred with lower stroke volume and cardiac output. The present findings provide new evidence for the acute modulatory influence of exercise on the cardiovascular responses to mental stress.

\section{Acknowledgments}

The authors thank Alzira Almeida de Lima (in memoriam), Renata Frauches Medeiros, Felipe de Sá Pereira, Thales Coelho Barbosa, Rogério Barbosa Magalhães Barros, and Tatiane Marinz de Souza for their assistance during the experiments, and Labs D'Or (Niterói, RJ, Brazil) for biochemical blood analyses. Research supported by CNPq (\#301168/2006-7) and FAPERJ (\#E-26/100.461/2007). A.C.G. Carvalho, A.R.K. Sales, and J.D. Rocha were supported by scholarships from CNPq. F.J. Neves and N.G. Rocha were supported by CAPES.

exercise hypotension in humans. Exerc Sport Sci Rev 2001; 29: 65-70.

12. de Tarso V, Nakamura FY, Polito MD. Influence of recovery posture on blood pressure and heart rate after resistance exercises in normotensive subjects. $J$ Strength Cond Res 2009; 23: 2487-2492.

13. Gomes AP, Doederlein PM. A review on post-exercise hypotension in hypertensive individuals. Arq Bras Cardiol 2011; 96: e100-e109.

14. Polito MD, Farinatti PT. The effects of muscle mass and number of sets during resistance exercise on postexercise hypotension. J Strength Cond Res 2009; 23: 2351-2357.

15. Somers VK, Conway J, Coats A, Isea J, Sleight P. Postexercise hypotension is not sustained in normal and hypertensive humans. Hypertension 1991; 18: 211-215.

16. Brandao Rondon MU, Alves MJ, Braga AM, Teixeira OT, Barretto AC, Krieger EM, et al. Postexercise blood pressure reduction in elderly hypertensive patients. J Am Coll Cardiol 2002; 39: 676-682.

17. Boone JB Jr, Probst MM, Rogers MW, Berger R. Postexercise hypotension reduces cardiovascular responses to stress. J Hypertens 1993; 11: 449-453.

18. Brownley KA, Hinderliter AL, West SG, Girdler SS, Sherwood A, Light KC. Sympathoadrenergic mechanisms in reduced hemodynamic stress responses after exercise. Med Sci Sports Exerc 2003; 35: 978-986.

19. Hamer M, Taylor A, Steptoe A. The effect of acute aerobic exercise on stress related blood pressure responses: a systematic review and meta-analysis. Biol Psychol 2006; 71: 183-190.

20. Santaella DF, Araujo EA, Ortega KC, Tinucci T, Mion D Jr, Negrao CE, et al. Aftereffects of exercise and relaxation on blood pressure. Clin J Sport Med 2006; 16: 341-347.

21. Steptoe $A$, Vogele $C$. Methodology of mental stress testing in cardiovascular research. Circulation 1991; 83: II-14-II-24. 
22. Dujic Z, Ivancev V, Valic Z, Bakovic D, Marinovic-Terzic I, Eterovic D, et al. Postexercise hypotension in moderately trained athletes after maximal exercise. Med Sci Sports Exerc 2006; 38: 318-322.

23. Hamer M, Jones J, Boutcher SH. Acute exercise reduces vascular reactivity to mental challenge in offspring of hypertensive families. J Hypertens 2006; 24: 315-320.

24. Teixeira L, Ritti-Dias RM, Tinucci T, Mion JD, Forjaz CL. Post-concurrent exercise hemodynamics and cardiac autonomic modulation. Eur J Appl Physiol 2011; 111: 20692078.

25. Wesseling KH, Jansen JR, Settels JJ, Schreuder JJ. Computation of aortic flow from pressure in humans using a nonlinear, three-element model. J Appl Physiol 1993; 74: 2566-2573.

26. Guelen I, Westerhof BE, Van Der Sar GL, Van Montfrans GA, Kiemeneij F, Wesseling $\mathrm{KH}$, et al. Finometer, finger pressure measurements with the possibility to reconstruct brachial pressure. Blood Press Monit 2003; 8: 27-30.

27. Stroop JR. Studies of interference in serial verbal reactions. J Exp Psychol 1935; 18: 643-662.

28. Callister R, Suwarno NO, Seals DR. Sympathetic activity is influenced by task difficulty and stress perception during mental challenge in humans. J Physiol 1992; 454: 373387.

29. Floras JS, Senn BL. Absence of post exercise hypotension and sympathoinhibition in normal subjects: additional evidence for increased sympathetic outflow in borderline hypertension. Can J Cardiol 1991; 7: 253-258.

30. Bousquet-Santos K, Soares PP, Nobrega AC. Subacute effects of a maximal exercise bout on endothelium-mediated vasodilation in healthy subjects. Braz J Med Biol Res 2005;
38: 621-627.

31. Alderman BL, Arent SM, Landers DM, Rogers TJ. Aerobic exercise intensity and time of stressor administration influence cardiovascular responses to psychological stress. Psychophysiology 2007; 44: 759-766.

32. Medeiros RF, Silva BM, Neves FJ, Rocha NG, Sales AR, Nobrega AC. Impaired hemodynamic response to mental stress in subjects with prehypertension is improved after a single bout of maximal dynamic exercise. Clinics 2011; 66: 1523-1529.

33. Hanel B, Teunissen I, Rabol A, Warberg J, Secher NH. Restricted postexercise pulmonary diffusion capacity and central blood volume depletion. J Appl Physiol 1997; 83: $11-17$.

34. Carroll D, Ring C, Hunt K, Ford G, Macintyre S. Blood pressure reactions to stress and the prediction of future blood pressure: effects of sex, age, and socioeconomic position. Psychosom Med 2003; 65: 1058-1064.

35. Loures DL, Sant Anna I, Baldotto CS, Sousa EB, Nobrega AC. [Mental stress and cardiovascular system]. Arq Bras Cardiol 2002; 78: 525-530.

36. Blumenthal JA, Fredrikson M, Kuhn CM, Ulmer RL, WalshRiddle M, Appelbaum M. Aerobic exercise reduces levels of cardiovascular and sympathoadrenal responses to mental stress in subjects without prior evidence of myocardial ischemia. Am J Cardiol 1990; 65: 93-98.

37. ATS/ACCP Statement on cardiopulmonary exercise testing. Am J Respir Crit Care Med 2003; 167: 211-277.

38. Balady GJ, Arena R, Sietsema K, Myers J, Coke L, Fletcher GF, et al. Clinician's Guide to cardiopulmonary exercise testing in adults: a scientific statement from the American Heart Association. Circulation 2010; 122: 191-225. 